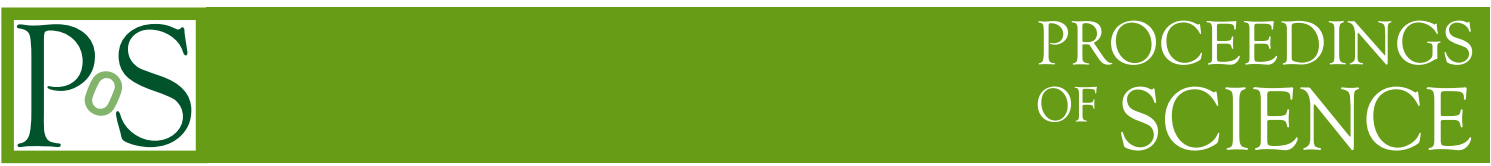

\title{
Probing Supersymmetry/BSM through Flavor Physics
}

\author{
Shaaban Khalil \\ Center for Fundamental Physics, Zewail City for Science and Technology, 6 October City, Cairo, \\ Egypt. \\ E-mail: skhalilezewailcity.edu.eg
}

Flavor Changing Neutral Current (FCNC) and Charge-Parity (CP) violating processes are a powerful tool to probe the New Physics (NP) beyond the Standard Model (SM). We show that supersymmetric contributions to the $b \rightarrow c \tau v_{\tau}$ transition can account simultaneously for a significant increase of both branching ratios of $\bar{B} \rightarrow D \tau \bar{v}_{\tau}$ and $\bar{B} \rightarrow D^{*} \tau \bar{v}_{\tau}$ with respect to the SM predictions, thereby approaching their experimentally measured values. We also show that in the Minimal Supersymmetric Sandard Model (MSSM) it is not possible to enhance $B R(h \rightarrow \tau \mu)$ without exceeding the experimental limits of the Lepton Flavor Violating (LFV) decays $\ell_{i} \rightarrow \ell_{j} \gamma$, while in Supersymmetric $B-L$ model with Inverse Seesaw (BLSSM-IS) the measured values of $B R(h \rightarrow \tau \mu)$ can be accommodated in a wide region of parameter space without violating LFV constraints. Thus, confirming the LFV Higgs decay results will be a clear signal of non-minimal Supersymmetric models.

38th International Conference on High Energy Physics 3-10 August 2016

Chicago, USA 


\section{Introduction}

Despite the great success of the SM in accounting for all the existing experimental data, compelling arguments indicate that it cannot be the complete theory of Nature. Among the theoretical problems that face the SM and strongly suggest NP beyond the SM are the following. The SM does not include gravity, therefore it cannot be valid at energy scales above $M_{P l} \sim 10^{19} \mathrm{GeV}$. Also the SM does not allow for neutrino masses, therefore it cannot be even valid at energy scales above $M_{\text {seesaw }} \sim 10^{15} \mathrm{GeV}$. Moreover, the fine-tuning problem of the Higgs mass and the puzzle of the dark matter suggest that the scale where the SM is replaced with a more fundamental theory is actually much lower, NP $\sim \mathscr{O}(1) \mathrm{TeV}$. In addition, the gauge couplings unification requires NP (in particular Supersymmetry (SUSY)) close to EW scale. It is tempting therefore to conclude that the SM is only an effective low energy limit of a more fundamental underlying theory.

FCNC and CP violation are ideal places to get indirect evidence of NP. In the SM, there are no FCNC processes at the tree level and they are strongly suppressed by Glashow-Illiopoulos-Maiani (GIM) mechanism and also by the small Cabibbo-Koboyashi-Maskawa (CKM) mixing angles at the loop level. It is also well established that the strength of the SM CP violation can not generate the observed size of the baryon asymmetry of the universe, and a new source of $\mathrm{CP}$ violation beyond the phase of the CKM mixing matrix is needed. The origin of flavor and CP violation is one of the most profound open questions in particle physics. Most extensions of the SM include new sources of flavor and CP violation. However, so far, most of experimental results on flavor observables are consistent with SM expectations and lead to strong indirect constraints on NP models. Increasing the sensitivity of flavor experiments at (Super) $B$-factories (BaBar and Belle) and LHCb maybe detect an indirect NP signal. It is worth mentioning that the charm and top quarks were first "seen" not by producing and observing them as "real" or physical particles but, rather, via their effects in FCNC process in $K$ and $B$ physics respectively.

Furthermore, the SM predicts that there should be no tree-level LFV Higgs coupling at the renormalizable level. These LFV processes are forbidden by the lepton flavor symmetry, which emerges accidentally in the SM. Nevertheless, many extensions of the SM do not exhibit such symmetries, and therefore, the measurements on the LFV processes can provide an indirect signal for the NP beyond the SM, in particular SUSY models. In the MSSM framework, even if one assumes no mixing in the lepton sector, a misalignment in the slepton sector with the soft SUSY breaking terms can induce LFV processes through the loop processes mediated by charginos or neutralinos. However, SUSY models with non-zero family mixing in the sleptons also result in enhancement in other LFV processes such as $\mu \rightarrow e \gamma, \tau \rightarrow e \gamma$, and $\tau \rightarrow \mu \gamma$. The experimental exclusion limits on these processes are established as $B R(\mu \rightarrow e \gamma) \leq 5.7 \times 10^{-13}$ [1], $B R(\tau \rightarrow$ $e \gamma) \leq 3.3 \times 10^{-8}$, and $B R(\tau \rightarrow \mu \gamma) \leq 4.4 \times 10^{-8}$ [2]. These measurements, especially those on $\mu \rightarrow e \gamma$, provide severe constraints on these models, that may lead to a sizable enhancement in LFV Higgs decays.

In this article we analyze the SUSY contributions to two processes that have been recently investigated by several collaborations, namely the semileptonic $B \rightarrow D \tau \nu$ and $h \rightarrow \tau \mu$ decay [3, 4]. We argue that SUSY contributions of the the MSSM, with non-universal soft breaking terms. might explain the discrepancy between the experimental results for $\bar{B} \rightarrow D \tau \nu_{\tau}$ and $\bar{B} \rightarrow D^{*} \tau \nu_{\tau}$ and the corresponding SM expectations. However, it is not possible to enhance $B R(h \rightarrow \tau \mu)$ in the MSSM 
without violating the experimental bound on the $B R(\tau \rightarrow \mu \gamma)$. We emphasize that in BLSSM-IS the measured values of $B R(h \rightarrow \tau \mu)$ can be accommodated in a wide region of parameter space without violating LFV constraints.

\section{2. $\bar{B} \rightarrow D \tau \nu_{\tau}$ and $\bar{B} \rightarrow D^{*} \tau \nu_{\tau}$ puzzle}

It has been recently reported a deviation from the SM expectations in the ratios

$$
\mathscr{R}(D)=\frac{\mathrm{BR}\left(\bar{B} \rightarrow D \tau \bar{v}_{\tau}\right)}{\operatorname{BR}\left(\bar{B} \rightarrow D l \bar{v}_{l}\right)}, \mathscr{R}\left(D^{*}\right)=\frac{\mathrm{BR}\left(\bar{B} \rightarrow D^{*} \tau \bar{v}_{\tau}\right)}{\operatorname{BR}\left(\bar{B} \rightarrow D^{*} l \bar{v}_{l}\right)},
$$

where, here, $l$ refers to either electron or muon. In particular, the Belle collaboration measured $[5,6]$

$$
\begin{aligned}
\mathscr{R}(D)^{\text {Belle }} & =0.375 \pm 0.064, \\
\mathscr{R}\left(D^{*}\right)^{\text {Belle }} & =0.302 \pm 0.030 \pm 0.011,
\end{aligned}
$$

whereas the results by the BaBar collaboration are [7]

$$
\begin{aligned}
\mathscr{R}(D)^{\mathrm{BaBar}} & =0.440 \pm 0.072, \\
\mathscr{R}\left(D^{*}\right)^{\mathrm{BaBar}} & =0.332 \pm 0.030 .
\end{aligned}
$$

In addition, the $\mathrm{LHCb}$ collaboration has found $\mathscr{R}\left(D^{*}\right)^{\mathrm{LHCb}}=0.336 \pm 0.027 \pm 0.030$ [8]. The SM predictions for $\mathscr{R}(D)$ and $\mathscr{R}\left(D^{*}\right)$ are $[9,10]$ :

$$
\begin{gathered}
\mathscr{R}(D)^{\mathrm{SM}}=0.305 \pm 0.012, \\
\mathscr{R}\left(D^{*}\right)^{\mathrm{SM}}=0.252 \pm 0.004
\end{gathered}
$$

which deviate by $\sim 1.7 \sigma$ from the combined experimental result for $\mathscr{R}(D)$ and $\sim 3 \sigma$ from the one for $\mathscr{R}\left(D^{*}\right)$. In addition, the combined four results disagree with the SM expectations at the $\sim 3.9 \sigma$ level. These deviations, if confirmed, could be important hints for NP, especially because the SM results for $\mathscr{R}(D)$ and $\mathscr{R}\left(D^{*}\right)$ are essentially independent of the parameterisation of the hadronic matrix elements.

The effective Hamiltonian for $b \rightarrow c l \bar{v}_{l}$ is

$$
\begin{aligned}
\mathscr{H}_{\mathrm{eff}} & =\frac{4 G_{F} V_{c b}}{\sqrt{2}}\left[\left(1+g_{V L}\right)\left[\bar{c} \gamma_{\mu} P_{L} b\right]\left[\bar{l} \gamma_{\mu} P_{L} v_{l}\right]+g_{V R}\left[\bar{c} \gamma_{\mu} P_{R} b\right]\left[\bar{l} \gamma_{\mu} P_{L} v_{l}\right]+g_{S L}\left[\bar{c} P_{L} b\right]\left[\bar{l} P_{L} v_{l}\right]\right. \\
& \left.+g_{S R}\left[\bar{c} P_{R} b\right]\left[\bar{l} P_{L} v_{l}\right]+g_{T}\left[\bar{c} \sigma^{\mu v_{\tau}} P_{L} b\right]\left[\bar{l} \sigma_{\mu v} P_{L} v_{l}\right]\right]
\end{aligned}
$$

where $G_{F}$ is the Fermi coupling constant, $V_{c b}$ is the CKM matrix element between charm and bottom quarks while $P_{L / R}=\left(1-/+\gamma_{5}\right) / 2$. Finally, $g_{i}$ is defined in terms of the Wilson coefficients $C_{i}$ as $g_{i}=C_{i}^{\mathrm{SUSY}} / C^{\mathrm{SM}}$, with $C^{\mathrm{SM}}=\frac{4 G_{F} V_{c b}}{\sqrt{2}}$. The amplitudes of possible NP contributions to $\bar{B} \rightarrow$ $D^{(*)} l \bar{v}_{l}, \mathscr{M} \equiv\left\langle D^{(*)} l \bar{v}_{l}\left|\mathscr{H}_{\text {eff }}\right| \bar{B}\right\rangle$, can be found in Ref.[11, 12]. Eventually, one can define both obsevables $\mathscr{R}(D)$ and $\mathscr{R}\left(D^{*}\right)$ as follows

$$
\mathscr{R}(D)=\frac{\Gamma\left(\bar{B} \rightarrow D \tau v_{\tau}\right)}{\Gamma\left(\bar{B} \rightarrow D l v_{l}\right)}, \quad \mathscr{R}\left(D^{*}\right)=\frac{\Gamma\left(\bar{B} \rightarrow D^{*} \tau v_{\tau}\right)}{\Gamma\left(\bar{B} \rightarrow D^{*} l v_{l}\right)} .
$$


Using the explicit formulae of the hadronic and leptonic amplitudes in Refs. $[9,10]$ (when the $l$ contribution is assumed to be described by the SM) and upon fixing the SM parameters and the form factors involved in the definition of the matrix elements to their central values as in Ref. [7], we can cast the explicit dependence of $\mathscr{R}(D)$ and $\mathscr{R}\left(D^{*}\right)$ upon the Wilson coefficients in the MSSM as follows:

$$
\begin{aligned}
\mathscr{R}(D) & =\mathscr{R}(D)^{\mathrm{SM}}\left[0.981\left|g_{S R}+g_{S L}\right|^{2}+\left|1+g_{V L}+g_{V R}\right|^{2}+0.811\left|g_{T}\right|^{2}\right. \\
& \left.+1.465 \operatorname{Re}\left[\left(1+g_{V L}+g_{V R}\right)\left(g_{S R}+g_{S L}\right)^{*}\right]+1.074 \operatorname{Re}\left[\left(1+g_{V L}+g_{V R}\right) g_{T}^{*}\right]\right], \\
\mathscr{R}\left(D^{*}\right) & =\mathscr{R}\left(D^{*}\right)^{\mathrm{SM}}\left[0.025\left|g_{S R}-g_{S L}\right|^{2}+\left|1+g_{V L}\right|^{2}+\left|g_{V R}\right|^{2}+16.739\left|g_{T}\right|^{2}\right. \\
& +0.094 \operatorname{Re}\left[\left(1+g_{V L}+g_{V R}\right)\left(g_{S R}-g_{S L}\right)^{*}\right]+6.513 \operatorname{Re}\left[g_{V R} g_{T}^{\tau *}\right] \\
& \left.-4.457 \operatorname{Re}\left[\left(1+g_{V L}\right) g_{T}^{*}\right]-1.748 \operatorname{Re}\left[\left(1+g_{V L}\right) g_{V R}^{*}\right]\right]
\end{aligned}
$$

From the above expressions one can easily see that, since $g_{i}<1$, the leading contributions to $\mathscr{R}(D)$ and $\mathscr{R}\left(D^{*}\right)$ are

$$
\begin{aligned}
\mathscr{R}(D) & =\mathscr{R}(D)^{\mathrm{SM}}\left(1+2 \operatorname{Re}\left[g_{V L}+g_{V R}\right]+1.465 \operatorname{Re}\left[\left(g_{S R}+g_{S L}\right)^{*}\right]+1.074 \operatorname{Re}\left[g_{T}^{*}\right]\right), \\
\mathscr{R}\left(D^{*}\right) & =\mathscr{R}\left(D^{*}\right)^{\mathrm{SM}}\left(1+2 \operatorname{Re}\left[g_{V L}\right]+0.094 \operatorname{Re}\left[\left(g_{S R}-g_{S L}\right)^{*}\right]-4.457 \operatorname{Re}\left[g_{T}^{*}\right]-1.748 \operatorname{Re}\left[g_{V R}^{*}\right]\right) .
\end{aligned}
$$

Thus, in case of a dominant scalar contribution (and negligible vector and tensor ones), it is clear that $\mathscr{R}\left(D^{*}\right)$ cannot be significantly larger than the SM expectation unless $g_{S R}-g_{S L}$ is larger than one (i.e., $C_{S}^{\mathrm{SUSY}}>C^{\mathrm{SM}}$ ), which is not possible. Recall that $g_{S R}$ is larger than $g_{S L}$ and it receives a contribution at the tree-level via charged Higgs exchange that yields

$$
g_{S R}=\frac{-m_{b} m_{\tau} \tan \beta^{2}}{2 \sqrt{2} G_{F} V_{c b} m_{H^{ \pm}}^{2}},
$$

where $m_{H^{ \pm}}$is the mass of the charged Higgs boson and $\tan \beta$ the ratio of the two vacuum expectation values. In Fig. 1 we display the regions in the $\left(g_{S L}, g_{S R}\right)$ plane that can accommodate the experimental results of $\mathscr{R}(D)$ and $\mathscr{R}\left(D^{*}\right)$ within a $1 \sigma$ confidence level for BaBar and Belle. We also show how $g_{S L}$ and $g_{S R}$ correlate at one-loop level. It is clear that the scalar contribution alone cannot account for both $\mathscr{R}(D)$ and $\mathscr{R}\left(D^{*}\right)$ simultaneously.

For a dominant vector contribution, one gets $\mathscr{R}(D) \sim 0.4$ and $\mathscr{R}\left(D^{*}\right) \sim 0.3$ if $g_{V L} \sim 0.13$ and $g_{V R} \sim 0.035$, which, as we will see, are quite plausible values. Finally, the tensor contribution, which is typically quite small, may affect only $\mathscr{R}\left(D^{*}\right)$. The SUSY contributions to $g_{V L}$ are generated from the penguin corrections to the vertices $b c W^{ \pm}$and $l v_{l} W^{ \pm}(l=e, \mu, \tau)$ through the exchange of charginos, neutralinos alongside squarks and sleptons/sneutrinos, respectively. However, one should note that the enhancement of the $b c W^{ \pm}$penguin will affect the $\operatorname{BR}\left(B \rightarrow D^{(*)} l v_{l}\right)$, which is very consistent with the SM results. Therefore, we adopt a scenario with heavy squarks, so that the quark penguins become negligible. Hence, we will focus on SUSY effects on the $l v_{l} W^{ \pm}$ $(l=e, \mu, \tau)$ penguins, which are displayed in Fig. 2.

In this scenario, one should consider a possible constraint due to the direct measurement of the $W$ boson decay width that leads to [13]

$$
\Gamma(W \rightarrow \tau v) / \Gamma(W \rightarrow e v)=1.063 \pm 0.027 .
$$



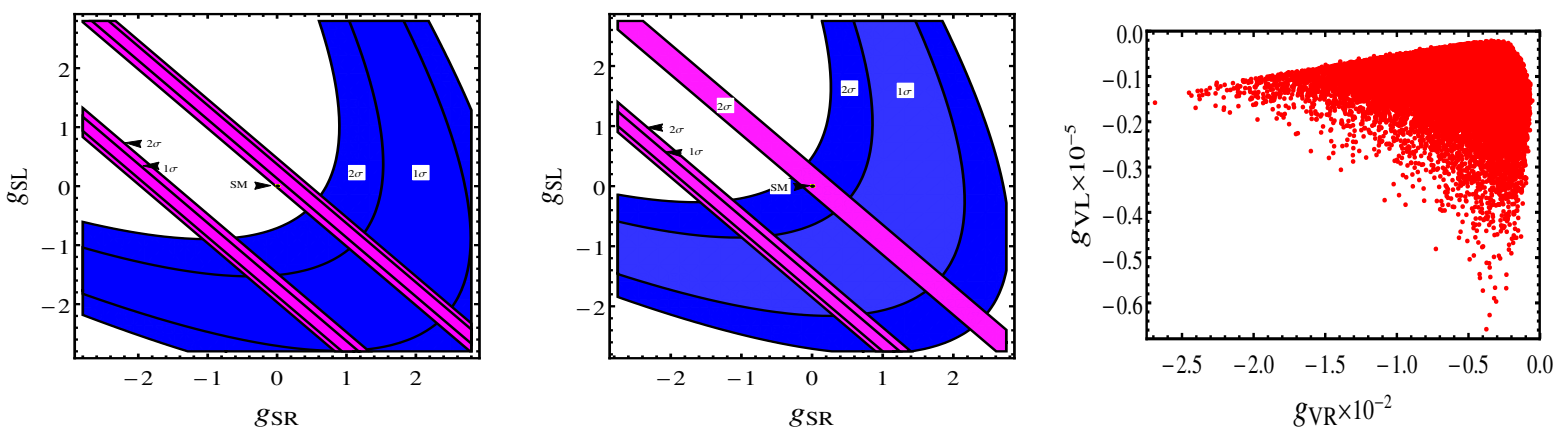

Figure 1: The allowed regions in the $\left(g_{S L}, g_{S R}\right)$ plane by the $1 \sigma$ and $2 \sigma$ experimental results on $\mathscr{R}(D)$ (magenta) and $\mathscr{R}\left(D^{*}\right)$ (blue) of BaBar (left) and Belle (middle). SM and complete SUSY predictions (tree, penguin and box contributions, where tree-level effect is the dominant for the scalar interactions) are also included and they coincide with the black point. The correlation between the SUSY corrected values of $g_{S L}$ and $g_{S R}$ is displayed in the right panel.

The SM prediction for this ratio is given by $\sim 0.999267$, which is consistent with the measured value. The decay width of $W \rightarrow \nmid v$ with SUSY contribution can be parametrised as

$$
\Gamma(W \rightarrow \nmid v)=\frac{G_{F} m_{W}^{3}}{6 \sqrt{2} \pi}\left(1-\frac{m_{l}^{2}}{m_{W}^{2}}\right)^{2}\left(1+\frac{1}{2} \frac{m_{l}^{2}}{m_{W}^{2}}\right)\left|1+g_{V L}^{\prime}\right|^{2} .
$$

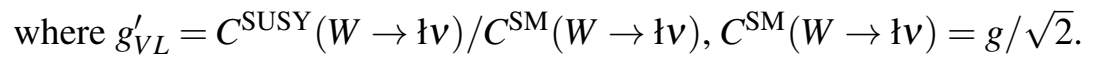

Another important constraint should be considered in this scenario which comes from $\tau$ decay. The universality of $\tau \rightarrow v_{\tau} l \nu_{l}$ [14], with $l=e, \mu$, given by

$$
\left(\frac{g_{\mu}}{g_{e}}\right)^{2}=\frac{B R\left(\tau \rightarrow \mu v_{\tau} v_{\mu}\right)}{B R\left(\tau \rightarrow e v_{\tau} v_{e}\right)} \frac{f\left(m_{e}^{2} / m_{\tau}^{2}\right)}{f\left(m_{\mu}^{2} / m_{\tau}^{2}\right)},
$$

where $f(x)=1-8 x+8 x^{3}-x^{4}-12 x^{2} \log (x)$. The universality of the gauge interaction in the SM leads $g_{\mu}=g_{e}$ and thus we can conclude the SM prediction for the decay width ratio

$$
\frac{\Gamma\left(\tau \rightarrow \mu v_{\tau} v_{\mu}\right)}{\Gamma\left(\tau \rightarrow e v_{\tau} v_{e}\right)}=\frac{f\left(m_{\mu}^{2} / m_{\tau}^{2}\right)}{f\left(m_{e}^{2} / m_{\tau}^{2}\right)}=0.9726
$$

the current experimental result [15] is $0.979 \pm 0.004$. For the SUSY contribution, the Eq.(22) can be written as

$$
\frac{\Gamma\left(\tau \rightarrow \mu v_{\tau} v_{\mu}\right)}{\Gamma\left(\tau \rightarrow e v_{\tau} v_{e}\right)}=0.9726 \frac{\left|1+g_{V L}^{\mu}\right|^{2}}{\left|1+g_{V L}^{e}\right|^{2}}
$$

where $g_{V L}^{l}=C^{\mathrm{SUSY}}\left(\tau \rightarrow v_{\tau} l v_{l}\right) / C^{\mathrm{SM}}\left(\tau \rightarrow v_{\tau} l v_{l}\right)$ with $C^{\mathrm{SM}}\left(\tau \rightarrow v_{\tau} l v_{l}\right)=2 \sqrt{2} G_{F}$. Here, our calculation is based on FlavorKit [16], SARAH [17] and SPheno [18], although the dominant penguin corrections were also derived analytically.

In Fig. 3 we display the regions in the $\left(g_{V L}, g_{V R}\right)$ plane that can accommodate the BaBar and Belle results on $\mathscr{R}(D)$ and $\mathscr{R}\left(D^{*}\right)$ within a $1 \sigma$ confidence level and compare these to the MSSM expectations at the one-loop level. It is clear that the contributions that induce vector operators, like the aforementioned triangle diagrams, lead to $\mathscr{R}(D)$ and $\mathscr{R}\left(D^{*}\right)$ close to or within the experimental regions. We can also conclude that $g_{V L}$ must be non-vanishing and of order 0.1 while $g_{V R}$ can be 


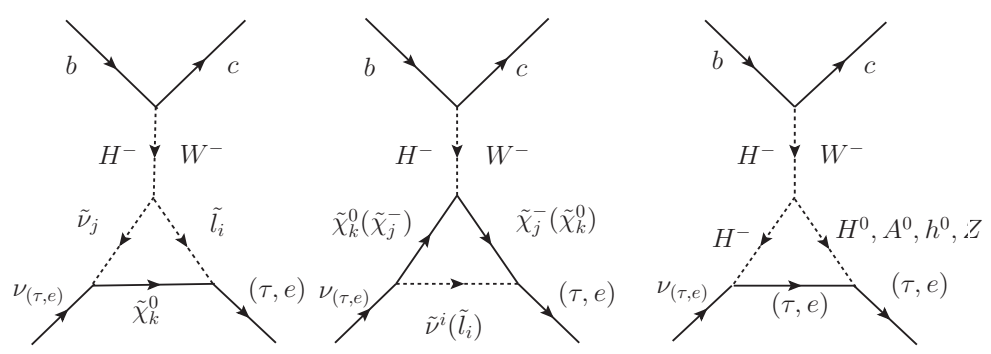

Figure 2: Triangle diagrams (penguins) contributing to, e.g., $b \rightarrow c(\tau, e) v_{(\tau, e)}$ affecting the leptonic vertex.
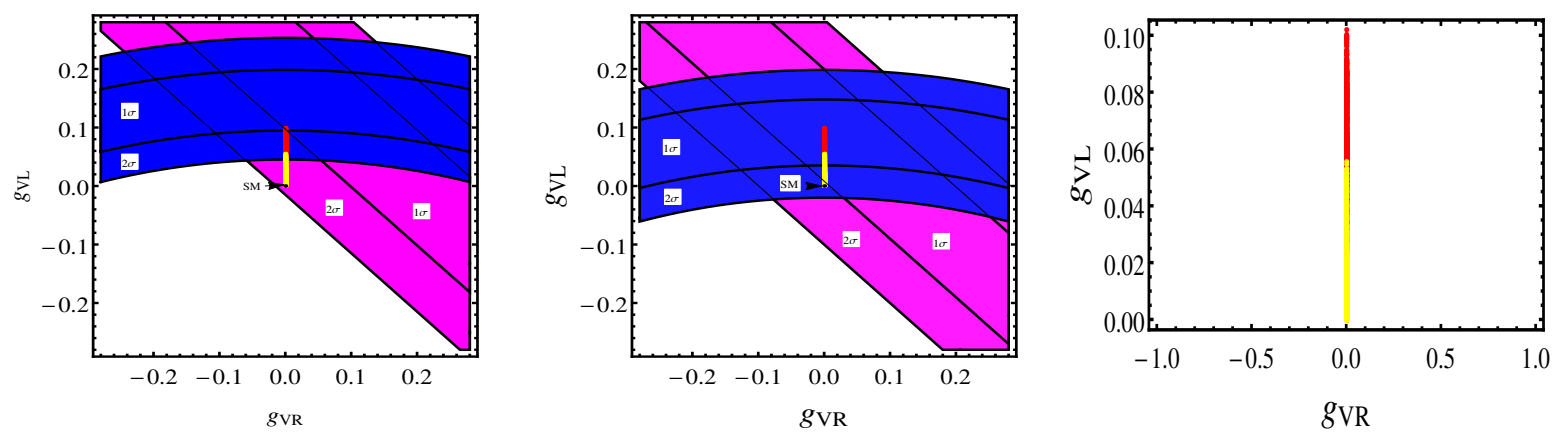

Figure 3: The allowed regions in the $\left(g_{V L}, g_{V R}\right)$ plane by the $1 \sigma$ and $2 \sigma$ experimental results on $\mathscr{R}(D)$ (magenta) and $\mathscr{R}\left(D^{*}\right)$ (blue) of BaBar (left) and Belle (middle). SM (black point) and complete SUSY (red points) predictions (penguin and box contributions, where the penguin is the dominant one) are also included. The correlation between the SUSY corrected values of $g_{V L}$ and $g_{V R}$ is displayed in the right panel, where yellow points represent the configurations that yield $\Gamma\left(\tau \rightarrow \mu v_{\tau} v_{\mu}\right) / \Gamma\left(\tau \rightarrow e v_{\tau} v_{e}\right)$ within experimental limits while the red ones are the complete sample.

in the range $[-0.1,0.1]$. Also, it is remarkable that our SUSY model can perfectly account for the Belle results and is within $2 \sigma$ of the BaBar ones.

Let us now try to decode our results, by concentrating on the Wilson coefficient $C_{V L}$, which sees contributions induced by the penguin topologies in Fig. 2. Firstly, we can confirm that the graph with neutral Higgs bosons is small (yet not negligible) while the other two are roughly comparable. Thus, the emerging $C_{V L}^{\mathrm{SUSY}}$ term is essentially $\left(h^{0} \equiv H_{\mathrm{SM}}\right)$

$$
C_{V L}^{\mathrm{SUSY}}=C_{V L}^{\tilde{\tau}}+C_{V L}^{\tilde{V}}+C_{V L}^{\left(A^{0}, H^{0}, h^{0}\right)} .
$$

One can show that the leading contributions to $C_{V L}$ are

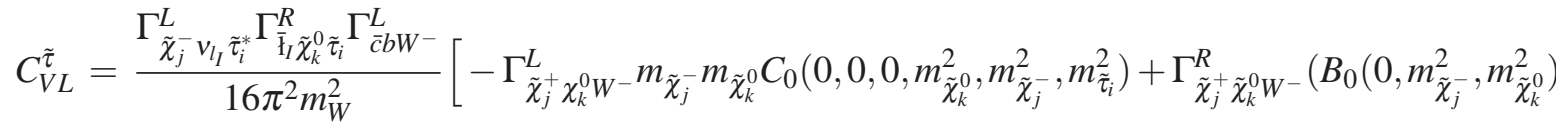

$$
\begin{aligned}
& \left.\left.-2 C_{00}\left(0,0,0, m_{\tilde{\chi}_{k}^{0}}^{2}, m_{\tilde{\chi}_{j}^{-}}^{2}, m_{\tilde{\tau}_{i}}^{2}\right)+m_{\tilde{\tau}_{i}}^{2} C_{0}\left(0,0,0, m_{\tilde{\chi}_{k}^{0}}^{2}, m_{\tilde{\chi}_{j}^{-}}^{2}, m_{\tilde{\tau}_{i}}^{2}\right)\right)\right], \\
& C_{V L}^{A^{0}}=\frac{2 \Gamma_{\overline{\mathrm{f}} v_{\mathfrak{1}} H^{-}}^{L} \Gamma_{\mathrm{i} \mathrm{i} A^{0}}^{R} \Gamma_{A^{0} H^{+} W^{-}} \Gamma_{\bar{c} b W^{-}}^{L}}{16 \pi^{2} m_{W}^{2}} C_{00}\left(0,0,0, m_{\mathfrak{1}}^{2}, m_{H^{-}}^{2}, m_{A^{0}}^{2}\right) .
\end{aligned}
$$

The wilson coefficient $C_{V L}^{\tilde{v}}$ can be obtained from $C_{V L}^{\tilde{\tau}}$ by exchanging $\Gamma_{\tilde{\chi}_{j}^{-} v_{l} \tilde{\tau}_{i}^{*}}^{L} \leftrightarrow \Gamma_{\tilde{\chi}_{j}^{-} \bar{l}_{l} \tilde{v}_{i}}^{R}, \Gamma_{\overline{\mathrm{i}}_{1}}^{R} \tilde{\chi}_{k}^{0} \tilde{\tau}_{i} \leftrightarrow$ 
$\Gamma_{v_{l} \tilde{\chi}_{k}^{0}{\tilde{v_{i}}}_{i}^{*}}^{L}$ and $m_{\tilde{\chi}_{k}^{0}} \leftrightarrow m_{\tilde{\chi}_{j}^{-}}$in the loop functions. The detailed expressions of the couplings and loop functions can be found in Ref. [3].
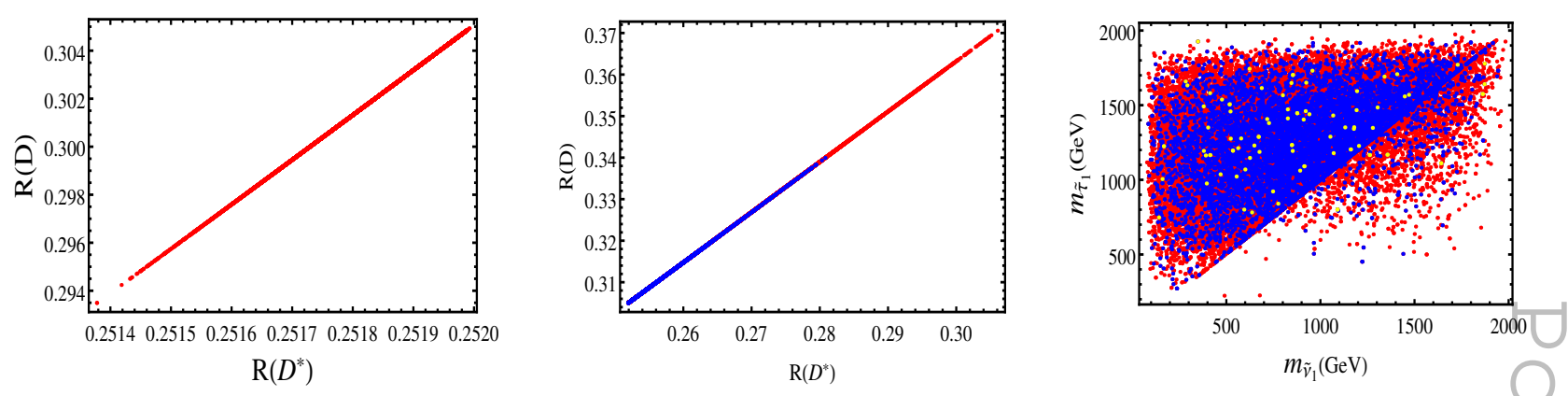

Figure 4: The correlation between $\mathscr{R}(D)$ and $\mathscr{R}\left(D^{*}\right)$ at tree level (left) and after the one-loop SUSY contributions through the lepton penguins (middle). The correlation between sneutrino and stau masses (right) for $\mathscr{R}(D)>0.33$. The color coding is the same as in previous figure.

Finally, the Wilson coefficients $C^{\mathrm{SUSY}}(W \rightarrow \nmid v)$ and $C^{\mathrm{SUSY}}\left(\tau \rightarrow v_{\tau} \nmid v_{l}\right)$ can be obtained from Eqs. (24) and (25) as follows

$$
\begin{gathered}
C^{\mathrm{SUSY}}\left(\tau \rightarrow v_{\tau} l v_{l}\right)=\frac{\Gamma_{\bar{v}_{\tau} \tau W^{-}}^{L}}{\Gamma_{\bar{c} b W^{-}}^{L}}\left(C_{V L}^{\tilde{\tau}}+C_{V L}^{\tilde{v}}+C_{V L}^{\left(A^{0}, H^{0}, h^{0}\right)}\right), \\
C^{\mathrm{SUSY}}(W \rightarrow \downarrow v)=\frac{m_{W}^{2}}{\Gamma_{\bar{c} b W^{-}}^{L}}\left(C_{V L}^{\tilde{\tau}}+C_{V L}^{\tilde{v}}+C_{V L}^{\left(A^{0}, H^{0}, h^{0}\right)}\right) .
\end{gathered}
$$

From these formulae one can notice that the loop functions of the decay $W \rightarrow \nmid v$ are suppressed with respect to the loop functions associated with $b \rightarrow c ł v$. In essence, the one-loop SUSY effects onto the $W$ widths are scaled by the $W$ squared mass while in $\mathscr{R}(D)$ and $\mathscr{R}\left(D^{*}\right)$ only by the meson squared masses. These suppressions are crucial for satisfying the experimental constraints on the ratio of the $W$ decay widths so that the results of $\mathscr{R}(D)$ and $\mathscr{R}\left(D^{*}\right)$ can be accommodated in unexcluded regions of the MSSM parameter space. It is also to be noted that these loop functions can be significantly enhanced if the chargino and neutralino masses are degenerate. Therefore, in our scan, we focus on the following ranges of the parameter space: the gaugino soft masses are given given by $M_{1}, M_{2} \in[110,500] \mathrm{GeV}$ and $M_{3}=1 \mathrm{TeV}$, the $\mu$ parameter $\in[100,500] \mathrm{GeV}, m_{A^{0}}^{2} \in$ $\left[0,25 \times 10^{4}\right](\mathrm{GeV})^{2}$, the $A$ terms $\in[-2000,-100] \mathrm{GeV}, M_{\tilde{Q}}, M_{\tilde{U}}$ and $M_{\tilde{D}}$ are fixed in the TeV range while the slepton soft mass terms $m_{\tilde{L}}$ and $m_{\tilde{E}} \in[100,2000] \mathrm{GeV}$. Finally, we take $\tan \beta \in[5,70]$.

In the left (middle) panel of Fig. 4 we present the correlation between $\mathscr{R}(D)$ and $\mathscr{R}\left(D^{*}\right)$ at tree-level (due to the SUSY contributions to the lepton penguins alone). As can be seen from this plot, in presence of MSSM one-loop corrections, $\mathscr{R}(D)$ can reach 0.354 while $\mathscr{R}\left(D^{*}\right)$ extends to 0.293, which are results rather consistent with the Belle measurements and not that far from the BaBar ones.

It is also very relevant to extract the typical mass spectra which are responsible for the MSSM configurations yielding $\mathscr{R}(D)$ and $\mathscr{R}\left(D^{*}\right)$ values (potentially) consistent with experimental measurements, as these might be accessible during Run 2 at the LHC. As an indication, this is done in Fig. 4 (right panel), limitedly to the lightest stau and sneutrino. The red points are all characterised by having both $\mathscr{R}(D)>\mathscr{R}(D)^{\mathrm{SM}}$ and $\mathscr{R}\left(D^{*}\right)>\mathscr{R}\left(D^{*}\right)^{\mathrm{SM}}$ whereas the blue and yellow 
ones are those compliant with the aforementioned $W$ width constraints, the yellow ones also being those with $\mathscr{R}(D)>0.33$. The plot shows a predilection of the highest $\mathscr{R}(D)$ and $\mathscr{R}\left(D^{*}\right)$ points for MSSM parameter configurations with $m_{\tilde{\tau}_{1}}>m_{\tilde{v}_{1}}$ while the absolute mass scale can cover the entire interval from $200 \mathrm{GeV}$ to $1 \mathrm{TeV}$. However, the points with $\mathscr{R}(D)>0.33$ require a rather large $\tilde{\tau}_{1}$ mass (say above $500 \mathrm{GeV}$ ) irrespectively of the $\tilde{v}_{1}$ one as well as large $\tan \beta$. This signals that there occurs an interplay between mass suppressions in the loops and enhancements in the couplings.

\section{Large $B R(h \rightarrow \tau \mu)$ in Supersymmetric Models}

The CMS and ATLAS collaborations reported the first signal of LFV Higgs decay $h \rightarrow \tau \mu$. The branching ratio of this decay is found as $[19,20]$

$$
\begin{aligned}
& B R(h \rightarrow \tau \mu)=\left(8.4_{-3.7}^{+3.9}\right) \times 10^{-3} \quad(\mathrm{CMS}) \\
& B R(h \rightarrow \tau \mu)=(7.7 \pm 6.2) \times 10^{-3} \quad(\text { ATLAS })
\end{aligned}
$$

As mentioned in the introduction, these LFV processes are forbidden in the SM. In the MSSM, the LFV decay $h \rightarrow \tau \mu$ can occur at one loop level via mediation of neutralinos or charginos [?] as shown in Figure 5. We assume that the LFV decay $h \rightarrow \tau \mu$ occurs at one-loop level, and the decay
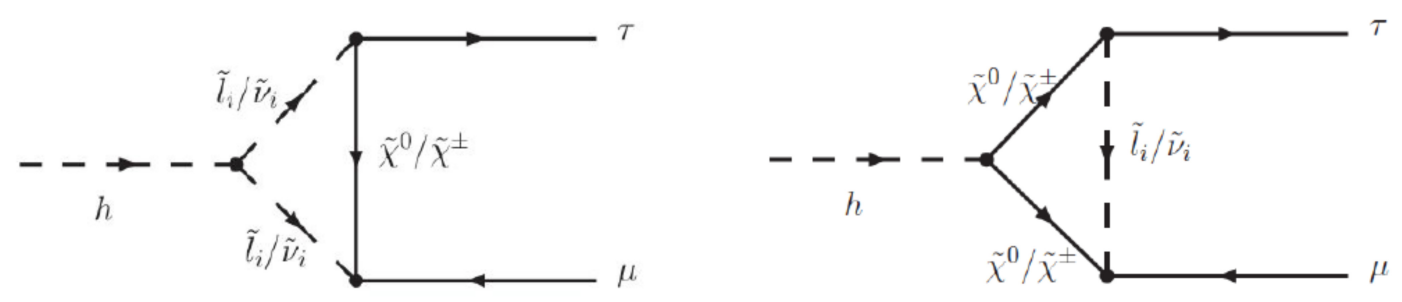

Figure 5: $h \rightarrow \tau \mu$ in the MSSM through chargino and sneutrino or neutralino and charged slepton exchanges

rate can be written as $\Gamma(h \rightarrow \tau \mu)=\Gamma(h \rightarrow \bar{\tau} \mu)+\Gamma(h \rightarrow \tau \bar{\mu})[21]$, where

$$
\begin{aligned}
\Gamma(h \rightarrow \tau \mu) & =\frac{1}{16 \pi M_{h}}\left[\left(1-\left(\frac{M_{\tau}+M_{\mu}}{M_{h}}\right)^{2}\right)\left(1-\left(\frac{M_{\tau}-M_{\mu}}{M_{h}}\right)^{2}\right)\right]^{1 / 2} \\
& \times\left[\left(M_{h}^{2}-M_{\tau}^{2}-M_{\mu}^{2}\right)\left(\left|F_{L}\right|^{2}+\left|F_{R}\right|^{2}\right)-4 M_{\tau} M_{\mu} \operatorname{Re}\left(F_{L} F_{R}^{*}\right)\right],
\end{aligned}
$$

and $F_{L}=F_{L}^{\chi^{+} \chi^{-\tilde{v}}}+F_{L}^{\chi^{0} \tilde{\ell}}+F_{L}^{\tilde{v} \tilde{v} \chi^{ \pm}}+F_{L}^{\chi^{0} \chi^{0} \tilde{\ell}}$, where these loop functions can be found in Ref.[4]. In the MSSM, the interaction vertices chargino (neutralino), leptopn and sneutrion (slepton) can be enhanced with non-universal slepton mass matrix and bino like neutralino. In addition, the trilinear scalar interaction coupling $T_{\ell}$ and bilinear Higgs mixing term $\mu$ may enhance the relevant couplings of Higgs. We perform two different scans over the following parameters to analyze the contributions from the sources mentioned above separately:

- Diagonal $T$-term: $0 \leq m_{\tilde{\mu}_{L, R}}, m_{\tilde{\tau}_{L, R}}, m_{L L}(2,3), M_{i} \leq 5 \mathrm{TeV}, 0 \leq \tan \beta \leq 60,\left|T_{l}\right| \leq 15 \mathrm{TeV}$.

- Diagonal slepton mass matrix: $0 \leq m_{\tilde{\mu}_{L, R}}, m_{\tilde{\tau}_{L, R}}, M_{i} \leq 5 \mathrm{TeV},\left|T_{l}(2,3)\right|,\left|T_{l}(3,2)\right| \leq 15 \mathrm{TeV}$, $0 \leq \tan \beta \leq 60$. 
where $m_{\tilde{\mu}_{L, R}}, m_{\tilde{\tau}_{L, R}}$ are the SSB masses of smuon and stau, while $m_{L L}(2,3)$ stands for the offdiagonal element of slepton mass matrix, which mixes the smuon and stau. $M_{i}(i=1,2,3)$ are the SSB gaugino mass terms for $U(1)_{Y}, S U(2)_{L}$ and $S U(3)_{c}$ respectively. $T_{l}$ is the trilinear scalar interaction coupling, and $\tan \beta$ is the ratio of vacuum expectation values (VEVs) of the MSSM Higgs doublets. In these scans, we employ SPheno [18] obtained by using SARAH [17]. Note that we require our solutions not to violate color and/or charge conservation at minima.
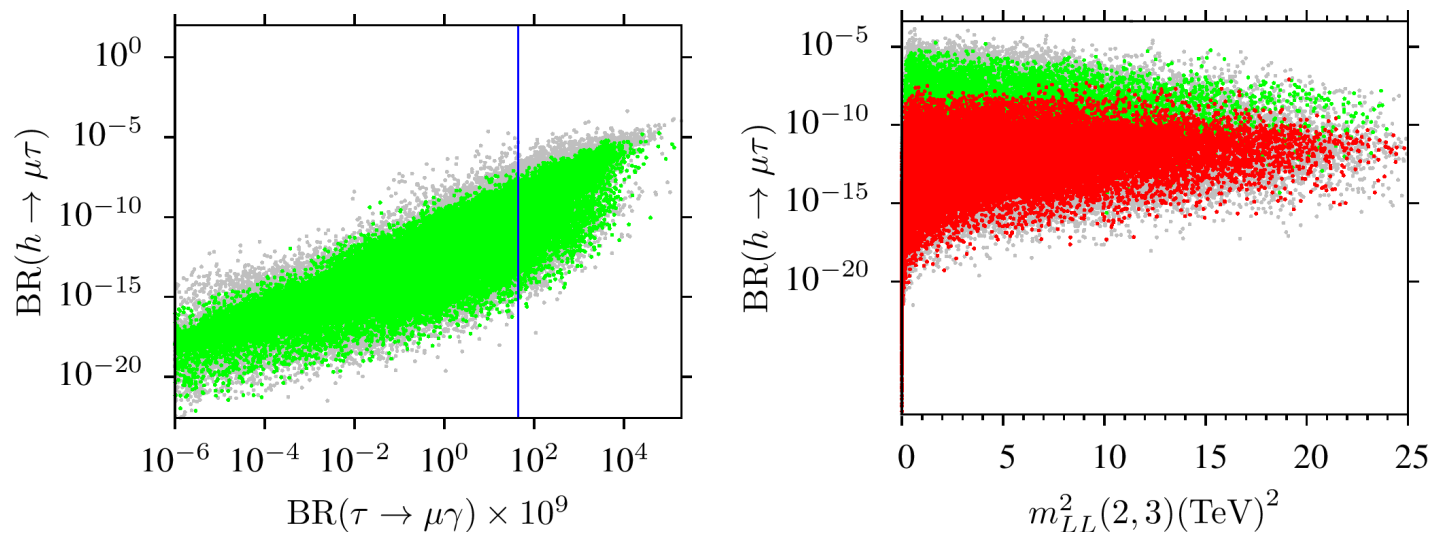

Figure 6: (Left) Correlation between $B R(h \rightarrow \tau \mu)$ and $B R(\tau \rightarrow \mu \gamma)$ in the MSSM. (Right) $B R(h \rightarrow \tau \mu)$ versus the slepton off-diagonal mass term $\left(m_{L L}^{\tilde{\ell}}\right)_{23}$ in the MSSM with non-diagonal slepton mass matrix. While gray points are excluded by the LHC constraints, green points satisfy the mass bounds on sparticles and the constraints from the rare B-meson decays. In addition, the vertical line in the left panel indicates the bound on $B R(\tau \rightarrow \mu \gamma)$, and the red points in the right panel form a subset of green and they satisfy the bound on $B R(\tau \rightarrow \mu \gamma)$.
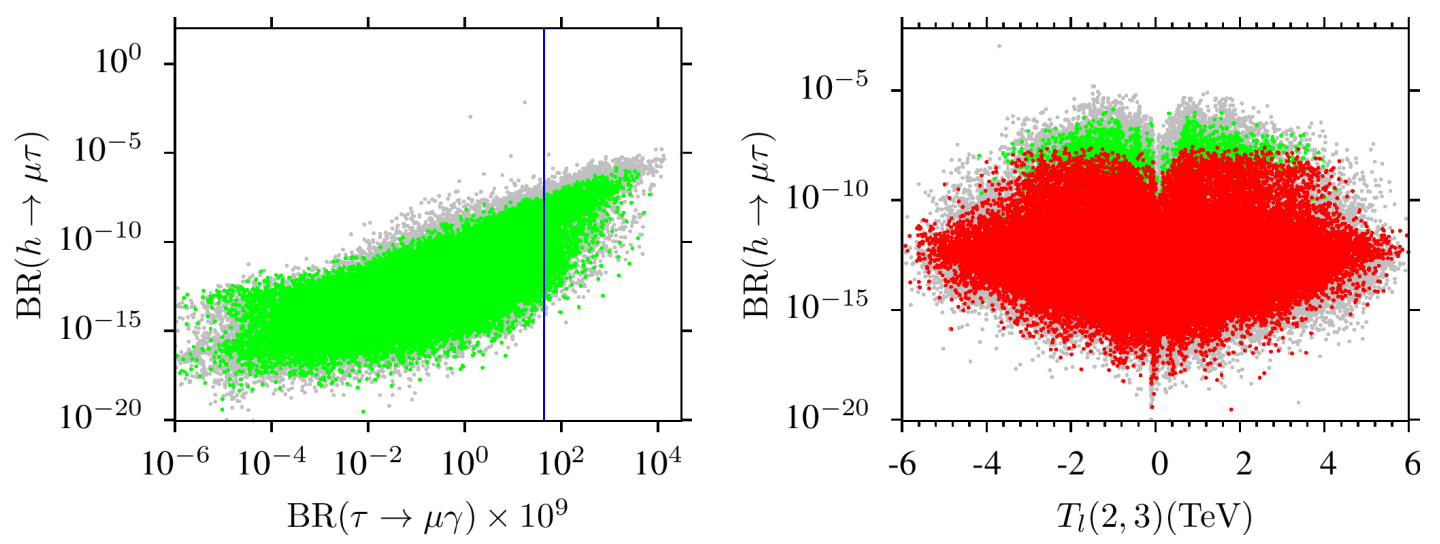

Figure 7: (Left) Correlation between $B R(h \rightarrow \tau \mu)$ and $B R(\tau \rightarrow \mu \gamma)$. (Right) $B R(h \rightarrow \tau \mu)$ versus the offdiagonal element of trilinear coupling $T_{23}^{\tilde{\ell}}$ in the MSSM with non-universal trilinear couplings. The color coding is the same as in the previous Figure .

Figure 6 displays the results from the scan with non-diagonal slepton mass matrix in terms of correlations between $B R(h \rightarrow \tau \mu)$ and $B R(\tau \rightarrow \mu \gamma)$, and also $B R(h \rightarrow \tau \mu)$ versus the slepton off-diagonal mass term $\left(m_{L L}^{\tilde{\ell}}\right)_{23}$. While gray points are excluded by the LHC constraints, green points satisfy the mass bounds on sparticles and the constraints from the rare B-meson decays. In addition, the vertical line in the left panel indicates the bound on $B R(\tau \rightarrow \mu \gamma)$, and the red points in 
the right panel form a subset of green and they satisfy the bound on $B R(\tau \rightarrow \mu \gamma)$. As seen from the left panel, $B R(h \rightarrow \tau \mu)$ can be as large as about $10^{-5}$ which is three magnitudes smaller than the values that can be probed, but this region violates the bound on $B R(\tau \rightarrow \mu \gamma)$. The maximum value for $B R(h \rightarrow \tau \mu)$ is about $10^{-8}$ without violating the bound on $B R(\tau \rightarrow \mu \gamma)$. Similar discussion can be followed for the results obtained for the MSSM with non-universal trilinear couplings, as shown in Figure 7. The color coding is the same as Figure 6. In conclusion, even though one can scan over a wider range of the parameters, it is not possible to enhance $B R(h \rightarrow \tau \mu)$ without violating the experimental bound on $B R(\tau \rightarrow \mu \gamma)$.

Now, we consider the minimal extension of the SM, based on the gauge group $S U(3)_{C} \times$ $S U(2)_{L} \times U(1)_{Y} \times U(1)_{B-L}$, which provides a suitable framework for the inverse seesaw mechanism, which can naturally account for light neutrino masses [?]. The particle content of supersymmetric version of this model (BLSSM-IS) includes the following superfields in addition to those in MSSM: (i) two SM singlet chiral Higgs superfields $\chi_{1,2}$ which are responsible for $U(1)_{B-L}$ breaking (ii) three sets of SM singlet chiral superfields, $v_{i}, s_{1_{i}}, s_{2_{i}}(i=1,2,3)$, to implement the IS mechanism, without generating $B-L$ anomaly. The Superpotential in this model is given by [22]

$$
W=-\mu^{\prime} \hat{\chi}_{1} \hat{\chi}_{2}+\mu \hat{H}_{u} \hat{H}_{d}+\mu_{S} \hat{s}_{2} \hat{s}_{2}-Y_{d} \hat{d} \hat{q} \hat{H}_{d}-Y_{\ell} \hat{e} \hat{l} \hat{H}_{d}+Y_{u} \hat{u} \hat{q} \hat{H}_{u}+Y_{s} \hat{v} \hat{\chi}_{1} \hat{s}_{2}+Y_{v} \hat{v} \hat{l} \hat{H}_{u} \cdot(
$$

In the BLSSM-IS, the coupling of charginos/neutralinos with sleptons/sneutrinos and also the Higgs coupling $\Gamma^{h \tilde{\ell}^{*}}$ remain very close to their values in the MSSM. However, the Higgs coupling with sneutrinos, $\Gamma^{h \tilde{v} \tilde{v}^{*}}$ is now given by

$$
\begin{aligned}
\Gamma^{h \tilde{v}_{i} \tilde{v}_{j}^{*}} & \sim-\sqrt{2} \mu \sum_{b=1}^{3} Z_{i b} \sum_{a=1}^{3} Y_{v, a b}^{*} Z_{j, 3+a}+\sqrt{2} \sum_{b=1}^{3} Z_{i b} \sum_{a=1}^{3} T_{v, a b}^{*} Z_{j, 3+a} \\
& +v_{1}^{\prime} \sum_{c=1}^{3} Z_{i c} \sum_{b=1}^{3} Z_{j, 6+b} \sum_{a=1}^{3} Y_{v, a b}^{*} Y_{s, a b}+\sqrt{2} \sum_{b=1}^{3} Z_{i 6+b} \sum_{a=1}^{3} T_{s, a b}^{*} Z_{j, 3+a}
\end{aligned}
$$

It is clear that this coupling can be significantly enhanced with large values of $\mu, T_{v}, T_{s}$ and $v_{1}^{\prime}$. This enhancement will enlarge the results of the decay width $\Gamma(h \rightarrow \tau v)$ with has no impact of the LFV process like $\mu \rightarrow e \gamma$ or $\tau \rightarrow \mu \gamma$. We perform a random scan over the following parameter space

$$
\begin{array}{cc}
0 \leq m_{0} & \leq 5(\mathrm{TeV}) \\
0 \leq \quad M_{1 / 2} & \leq 5(\mathrm{TeV}) \\
-3 \leq A_{0} / m_{0} & \leq 3 \\
1.2 \leq \tan \beta & \leq 60 \\
1 \leq \tan \beta^{\prime} & \leq 2
\end{array}
$$

$\mu_{S} \sim 10^{-7} \mathrm{GeV}$ and $M_{Z^{\prime}}=2.5 \mathrm{TeV}$ are fixed. In Figure 8 we present the results of BLSSM-IS for the $B R(h \rightarrow \tau \mu)$ versus $B R(\mu \rightarrow e \gamma)$, and also as function of $T_{v}, T_{s}$ and $\mu$. As usual, gray points are excluded by the LHC constraints, while the green points are allowed. The red points form a subset of green and they are consistent with the bound on $B R(\mu \rightarrow e \gamma)$. In contrast to the generic MSSM, $B R(h \rightarrow \tau \mu)$ does not exhibit an enhancement with the $B R(\mu \rightarrow e \gamma)$. This result can be explained by the fact that the the couplings of Higgs to the sneutrinos are enhanced with very large trilinear couplings and $\mu$ parameter. These couplings play a crucial role in generating $h \rightarrow \tau \mu$ decay at one loop level in BLSSM-IS, with no impact on the one loop contribution to $\ell_{i} \rightarrow \ell_{j} \gamma$. Hence, the 

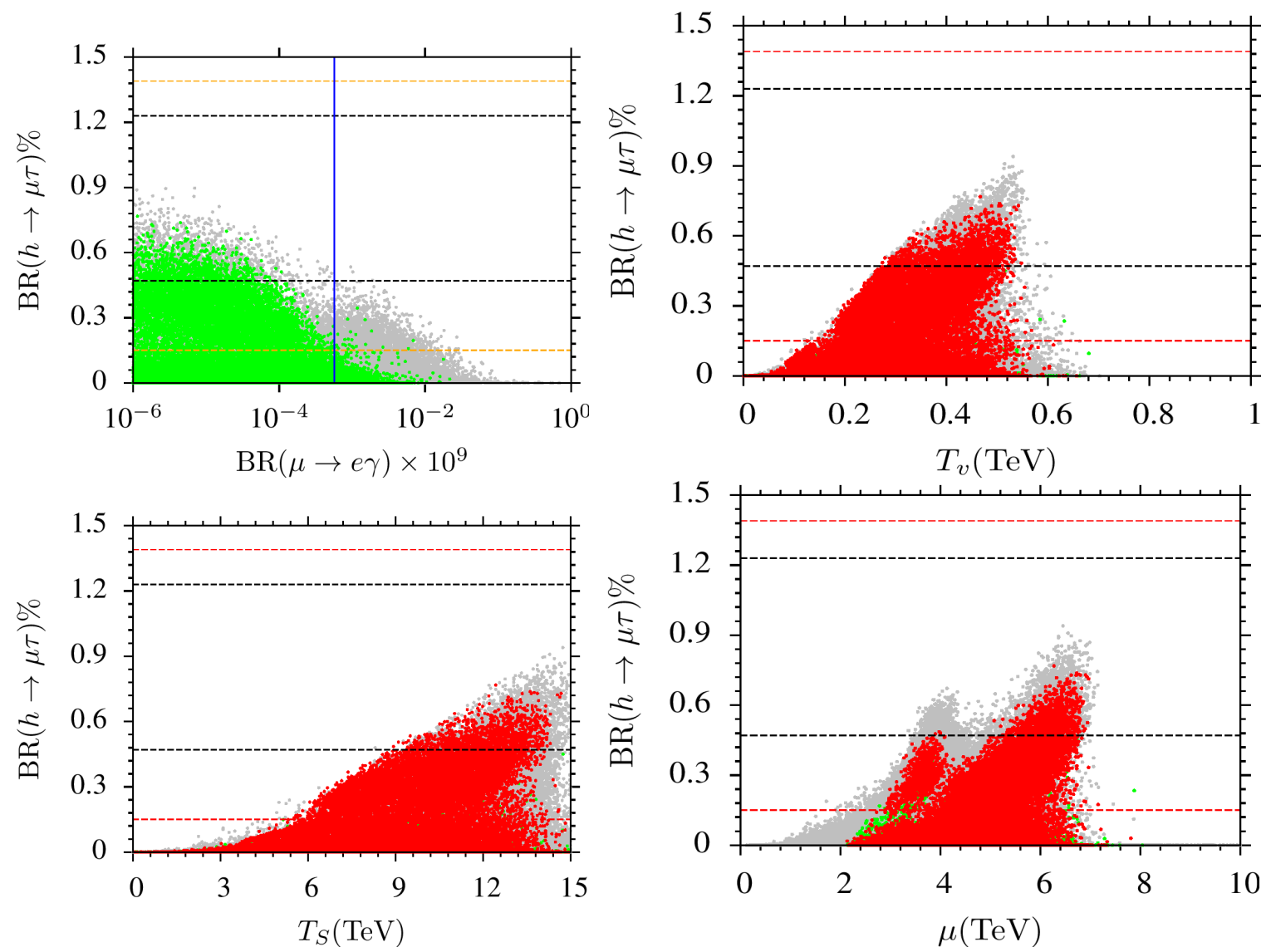

Figure 8: Plots in the $B R(h \rightarrow \tau \mu)-B R(\mu \rightarrow e \gamma), B R(h \rightarrow \tau \mu)-m_{0}, B R(h \rightarrow \tau \mu)-A_{0} / m_{0}$, and $B R(h \rightarrow$ $\tau \mu)-\tan \beta$ planes. Gray points are excluded by the LHC constraints, while the green points are allowed. The red points form a subset of green and they are consistent with the bound on $B R(\mu \rightarrow e \gamma)$. The black (orange) dashed lines indicate the best fit for the $\mathrm{BR}(h \rightarrow \mu \tau)$ obtained by CMS [19] (ATLAS [20]).

BLSSM-IS provides an example for a SUSY model with no correlation between $B R(h \rightarrow \tau \mu)$ and $B R\left(\ell_{i} \rightarrow \ell_{j} \gamma\right)$.

\section{Conclusion}

In this review I have emphasized that the MSSM has the potential to explain recent data produced by BaBar and Belle which revealed a rather significant excess above and beyond the best SM predictions available in the observed $\operatorname{BR}\left(\bar{B} \rightarrow D \tau \bar{v}_{\tau}\right)$ and $\mathrm{BR}\left(\bar{B} \rightarrow D^{*} \tau \bar{v}_{\tau}\right)$ relative to the light lepton cases. In addition, the LFV Higgs boson decay $h \rightarrow \tau \mu$ in the MSSM and BLSSM-IS has been analyzed. I showed that in the MSSM, $B R(h \rightarrow \tau \mu)$ can be realized at the order of $10^{-5}$, nevertheless, such solutions violate the constraints from another LFV process, $\tau \rightarrow \mu \gamma$. In the BLSSM-IS framework. $B R(h \rightarrow \tau \mu)$ can be as large as $0.77 \%$ without violating the LFV constraints including $\mu \rightarrow e \gamma$. This is because of the enhancemnet of the Higgs couplings with sneutrinos with large values of trilinear and $\mu$ parameters. 


\section{Acknowledgments}

I would like to thank my collaborators S. Moretti, D. Boubaa, C. Un, and A. Hammad. The work is partially supported by the STDF project 13858, the grant H2020-MSCA-RISE-2014 no. 645722 (NonMinimalHiggs), and the European Union's Horizon 2020 research and innovation programme under the Marie Curie grant agreement No 690575.

\section{References}

[1] J. Adam et al. [MEG Collaboration], Phys. Rev. Lett. 110, 201801 (2013) doi:10.1103/PhysRevLett.110.201801 [arXiv:1303.0754 [hep-ex]].

[2] B. Aubert et al. [BaBar Collaboration], Phys. Rev. Lett. 104, 021802 (2010) doi:10.1103/PhysRevLett.104.021802 [arXiv:0908.2381 [hep-ex]].

[3] D. Boubaa, S. Khalil and S. Moretti, arXiv:1604.03416 [hep-ph].

[4] A. Hammad, S. Khalil and C. S. Un, arXiv:1605.07567 [hep-ph].

[5] Belle Collaboration, Phys. Rev. D 92, 072014 (2015).

[6] Belle Collaboration, arXiv:1603.06711 [hep-ex].

[7] BaBar Collaboration, Phys. Rev. D 88, 072012 (2013).

[8] LHCb Collaboration, Phys. Rev. Lett. 115, 111803 (2015).

[9] M. Tanaka and R. Watanabe, Phys. Rev. D 87, 034028 (2013).

[10] Y. Sakaki, M. Tanaka, A. Tayduganov and R. Watanabe, Phys. Rev. D 88, 094012 (2013).

[11] K. Hagiwara, A. D. Martin and M. F. Wade, Nucl. Phys. B 327, 569 (1989).

[12] K. Hagiwara, A. D. Martin and M. F. Wade, Phys. Lett. B 228, 144 (1989).

[13] K. A. Olive et al. [Particle Data Group Collaboration], Chin. Phys. C 38, 090001 (2014).

[14] B. Aubert et al. [BaBar Collaboration], Phys. Rev. Lett. 105, 051602 (2010)

[15] C. Patrignani, Chin. Phys. C 40, no. 10, 100001 (2016).

[16] W. Porod, F. Staub and A. Vicente, Eur. Phys. J. C 74, 2992 (2014).

[17] F. Staub, Comput. Phys. Commun. 185, 1773 (2014).

[18] W. Porod and F. Staub, Comput. Phys. Commun. 183, 2458 (2012).

[19] V. Khachatryan et al. [CMS Collaboration], Phys. Lett. B 749, 337 (2015) doi:10.1016/j.physletb.2015.07.053 [arXiv:1502.07400 [hep-ex]].

[20] G. Aad et al. [ATLAS Collaboration], JHEP 1511, 211 (2015) doi:10.1007/JHEP11(2015)211 [arXiv:1508.03372 [hep-ex]]; G. Aad et al. [ATLAS Collaboration], JHEP 1511, 211 (2015) doi:10.1007/JHEP11(2015)211 [arXiv:1508.03372 [hep-ex]].

[21] E. Arganda, A. M. Curiel, M. J. Herrero and D. Temes, Phys. Rev. D 71, 035011 (2005) doi:10.1103/PhysRevD.71.035011 [hep-ph/0407302]; and references therein.

[22] A. Elsayed, S. Khalil and S. Moretti, Phys. Lett. B 715, 208 (2012) doi:10.1016/j.physletb.2012.07.066 [arXiv:1106.2130 [hep-ph]]. 